\title{
Queue Layouts of Planar 3-Trees
}

\author{
Jawaherul Md. Alam ${ }^{1}$ Michael A. Bekos² - Martin Gronemann ${ }^{3}$. \\ Michael Kaufmann ${ }^{2}$. Sergey Pupyrev ${ }^{1}$
}

Received: 6 October 2018 / Accepted: 6 March 2020 / Published online: 23 March 2020

(c) The Author(s) 2020

\begin{abstract}
A queue layout of a graph $G$ consists of a linear order of the vertices of $G$ and a partition of the edges of $G$ into queues, so that no two independent edges of the same queue are nested. The quеие number of graph $G$ is defined as the minimum number of queues required by any queue layout of $G$. In this paper, we continue the study of the queue number of planar 3-trees, which form a well-studied subclass of planar graphs. Prior to this work, it was known that the queue number of planar 3-trees is at most seven. In this work, we improve this upper bound to five. We also show that there exist planar 3-trees whose queue number is at least four. Notably, this is the first example of a planar graph with queue number greater than three.
\end{abstract}

Keywords Queue layouts · Constant queue number · Planar 3-trees

A preliminary version of this article has appeared in the proceedings of the 26th International Symposium on Graph Drawing and Network Visualization (GD 2018). This work is supported by the DFG Grant Ka812/17-1 and DAAD Project 57419183.

Michael A. Bekos

bekos@informatik.uni-tuebingen.de

Jawaherul Md. Alam

jawaherul@gmail.com

Martin Gronemann

gronemann@informatik.uni-koeln.de

Michael Kaufmann

mk@informatik.uni-tuebingen.de

Sergey Pupyrev

spupyrev@gmail.com

1 Department of Computer Science, University of Arizona, Tucson, USA

2 Institut für Informatik, Universität Tübingen, Tübingen, Germany

3 Institut für Informatik, Universität zu Köln, Köln, Germany 


\section{Introduction}

In a quеие layout [19], the vertices of a graph are restricted to a line and its edges are drawn at different half-planes delimited by this line, called queues. The task is to find a linear order of the vertices along the underlying line and a corresponding assignment of the edges of the graph to the queues, so that no two independent edges of the same queue are nested; see Fig. 1 for an illustration. Recall that two edges are called independent, if they do not share an endvertex. The queue number of a graph is the smallest number of queues that are required by any queue layout of the graph. Note that queue layouts form the "dual" concept of stack layouts [22] (widely known also as book embeddings), which do not allow two edges of the same stack to cross.

Apart from the intriguing theoretical interest, queue layouts find applications in several domains $[3,18,23,29]$. As a result, they have been studied extensively over the years $[5,8,13,17,19,24,25,27-30]$. The most remarkable result in this area is due to Dujmović et al. [9], who recently proved that planar graphs have constant queue number (by the time of writing at most 49) improving upon a series of results $[2,5,7]$ and thus settling in the positive an old conjecture by Heath, Leighton and Rosenberg [18]. Notably, this breakthrough result has several important implications, e.g., that (i), (ii) every $n$-vertex planar graph admits a $\mathcal{O}(1) \times \mathcal{O}(1) \times \mathcal{O}(n)$ straight-line grid drawing [31], (iii) every Hamiltonian bipartite planar graph admits a 2-layer drawing and an edge-coloring of bounded size, in which edges of the same color do not cross [12], and (iv) the queue number of $k$-planar graphs is also bounded by a constant for fixed values of $k$ [13].

Improved upper bounds on the queue number are known for several subclasses of planar graphs. Every tree has queue number one [19], outerplanar graphs have queue number at most two [18], and series-parallel graphs have queue number at most three [27]. Planar 3-trees have queue number at most seven [30], although they were conjectured to have super-constant queue number by Pemmaraju [24]; recall that a planar 3-tree is a triangulated plane graph $G$ with $n \geq 3$ vertices, such that $G$ is either a 3-cycle, if $n=3$, or has a vertex whose deletion gives a planar 3-tree with $n-1$ vertices, if $n>3$. As a matter of fact, every graph that admits a 1 -queue layout is planar with at most $2 n-3$ edges; however, testing this property is an $\mathcal{N P}$ -complete problem [18]; for a survey that also covers results for non-planar graphs the interested reader is referred to [13].

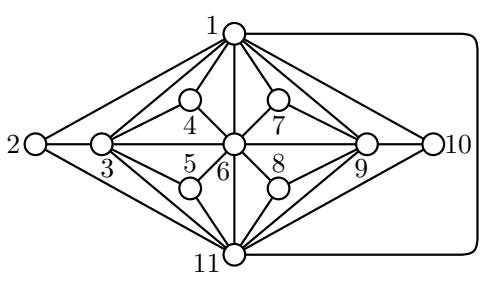

(a)

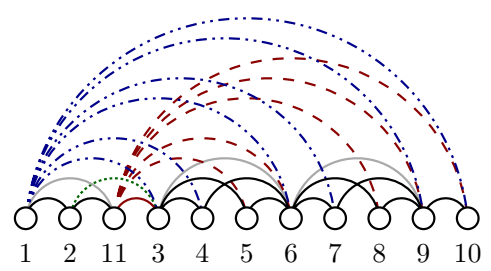

(b)

Fig. 1 a The Goldner-Harary planar 3-tree, and $\mathbf{b}$ a 5-queue layout of it produced by our algorithm, in which edges of different queues are colored differently 
Table 1 Queue numbers of various subclasses of planar graphs

\begin{tabular}{|c|c|c|c|c|}
\hline \multirow{2}{*}{ Graph class } & \multicolumn{2}{|c|}{ Upper bound } & \multicolumn{2}{|c|}{ Lower bound } \\
\hline & Old & New & Old & New \\
\hline Tree & 1 [19] & & 1 [19] & \\
\hline Outerplanar & $2[18]$ & & $2[19]$ & \\
\hline Series-parallel & $3[27]$ & & $3[30]$ & \\
\hline Planar 3-tree & $7[30]$ & 5 [Thm. 1] & $3[30]$ & 4 [Thm. 2] \\
\hline Planar & 49 [9] & & $3[30]$ & 4 [Thm. 2] \\
\hline
\end{tabular}

Our contribution. In this paper, we present improved upper and lower bounds on the queue number of planar 3-trees. More precisely, we first discuss in Sect. 2 known results that are useful for our work. In Sect. 3, we improve the upper bound on the queue number of planar 3-trees from seven [30] to five. In Sect. 4, we show that there exist planar 3-trees whose queue number is at least four, thus strengthening a corresponding result of Wiechert [30] for general (that is, not necessarily planar) 3-trees; we stress that our lower bound is also the best known for planar graphs. Table 1 puts our results in the context of existing bounds. In Sect. 5, we discuss implications of our results to the closely-related track layouts [11]. Our work leads to a number of interesting research questions, which we list in Sect. 6.

Remark The core of the approach introduced by Dujmović et al. [9] consists of partitioning the input planar graph into sets of vertices, called bags, such that the graph induced by the bags is planar and has treewidth at most 3 . The queue number of the input graph is then upper bounded by an expression that depends on the queue number of planar 3-trees. It is worth noting that the authors of [9] use our main result to obtain the bound on the queue number of planar graphs, as paper [9] appeared after the conference version [1] of this work.

\section{Preliminaries}

In this section, we present preliminary notions and notation that is used throughout this paper. We also present known results from the literature that are useful for our work. We assume familiarity with basic graph theoretic notions (see, e.g., [16]). Also, for standard definitions on planar graphs and drawings, we point the reader to $[4,20]$.

For a pair of distinct vertices $u$ and $v$ of a graph $G$, we write $u \prec v$ if $u$ precedes $v$ in a linear order of $G$. We also write $\left[v_{1}, v_{2}, \ldots, v_{k}\right]$ to denote that $v_{i}$ precedes $v_{i+1}$ for all $1 \leq i<k$. Let $F$ be a set of $k \geq 2$ independent edges $\left(s_{i}, t_{i}\right)$, that is, $F=\left\{\left(s_{i}, t_{i}\right) ; i=1,2, \ldots, k\right\}$. Assume without loss of generality that $s_{i} \prec t_{i}$, for all $1 \leq i \leq k$. If the linear order is $\left[s_{1}, \ldots, s_{k}, t_{k}, \ldots, t_{1}\right]$, then we say that $F$ is a $k$-rainbow, while if the linear order is $\left[s_{1}, \ldots, s_{k}, t_{1}, \ldots, s_{k}\right]$, we say that $F$ is a $k$-twist. 


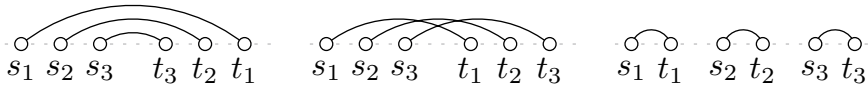

(a) (b)

Fig. 2 Illustration of: a a 3-rainbow, b a 3-twist, and $\mathbf{c}$ a 3-necklace

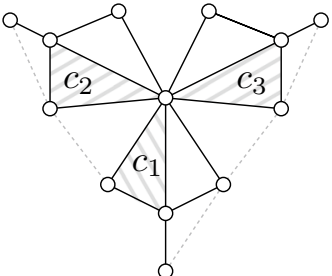

(a)

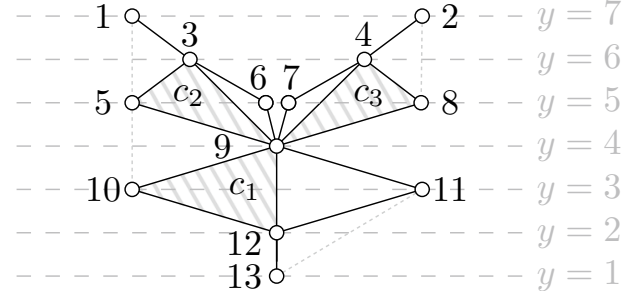

(b)

Fig. 3 Illustrations of: a an internally-triangulated outerplane graph $G_{0}$; the dotted-gray edges have been added to make it biconnected; its gray-shaded faces contain components $c_{1}, c_{2}$ and $c_{3}$ of the graph $G_{1}$ formally introduced Section 3; b the drawing $\Gamma\left(G_{0}\right)$ by Lemma 2; the vertex-labels indicate the linear order of its 2-queue layout; the anchor vertices of faces $\langle 9,10,12\rangle,\langle 3,5,9\rangle$ and $\langle 4,8,9\rangle$ are $10,5,8$, respectively

The edges of $F$ form a $k$-necklace, if $\left[s_{1}, t_{1}, \ldots, s_{k}, t_{k}\right]$; see Fig. 2. A preliminary result for queue layouts is the following.

Lemma 1 (Heath and Rosenberg [19]) A linear order of the vertices of a graph admits a $k$-queue layout if and only if there exists no $(k+1)$-rainbow.

Central in our approach is also the following construction by Dujmović et al. [11] for internally-triangulated outerplane graphs; for an illustration refer to Figs. 3a, b.

Lemma 2 (Dujmović et al. [11]) Every internally-triangulated outerplane graph $G$ admits a straight-line outerplanar drawing $\Gamma(G)$, such that the $y$-coordinates of the vertices of $G$ are integers, and the absolute value of the difference of the $y$-coordinates of the endvertices of each edge of $G$ is either one or two. Furthermore, the drawing can be used to construct a 2-queue layout of $G$.

Let $\langle u, v, w\rangle$ be a face of a drawing $\Gamma(G)$ produced by the constructive algorithm supporting Lemma 2 , where $G$ is an internally triangulated outerplane graph. Up to renaming of the vertices of this face, we may assume that $y(v)>y(w)$, $y(v)-y(u)=y(u)-y(w)=1$ and $y(v)-y(w)=2$. We refer to vertex $u$ as to the anchor vertex of the face $\langle u, v, w\rangle$ of $\Gamma(G)$. Vertices $v$ and $w$ are referred to as top and bottom, respectively. It is easy to verify that drawing $\Gamma(G)$ can be converted to a 2-queue layout of $G$ as follows: 
- for any two distinct vertices $u$ and $v$ of $G, u \prec v$ if and only if the $y$-coordinate of $u$ is strictly greater than the one of $v$, or the $y$-coordinate of $u$ is equal to the one of $v$ and $u$ is to the left of $v$ in $\Gamma(G)$,

- edge $(u, v)$ is assigned to the first (to the second) queue if and only if the absolute value of the difference of the $y$-coordinates of $u$ and $v$ is one (two, respectively) in $\Gamma(G)$.

In the following, we present interesting properties of the 2-queue layout produced from drawing $\Gamma(G)$. Let $\langle u, v, w\rangle$ and $\left\langle u^{\prime}, v^{\prime}, w^{\prime}\right\rangle$ be two distinct faces of $\Gamma(G)$, such that $u$ and $u^{\prime}$ are their anchors, $v$ and $v^{\prime}$ are their top vertices, and $w$ and $w^{\prime}$ are their bottom vertices.

Property 1 If $u \neq u^{\prime}$ and $u \prec u^{\prime}$ in the 2-queue layout, then $v \prec v^{\prime}\left(\right.$ if $\left.v \neq v^{\prime}\right)$ and $w \prec w^{\prime}\left(\right.$ if $\left.w \neq w^{\prime}\right)$.

Proof The property clearly holds if vertices $u$ and $u^{\prime}$ do not have the same $y$-coordinate. Otherwise, the property holds since $\Gamma(G)$ is planar.

Property 2 If $u=u^{\prime}, v \neq v^{\prime}$ and $v \prec v^{\prime}$ in the 2-queue layout, then $w \prec w^{\prime}$ (if $w \neq w^{\prime}$ ) .

Proof The property follows from the fact that $\Gamma(G)$ is planar.

Property 3 If $u=u^{\prime}, w \neq w^{\prime}$ and $w \prec w^{\prime}$ in the 2-queue layout, then $v \prec v^{\prime}$ (if $v \neq v^{\prime}$ ).

Proof The property follows from the fact that $\Gamma(G)$ is planar.

\section{The Upper Bound}

In this section, we prove that the queue number of every planar 3-tree is at most five. Our approach is inspired by the algorithm of Wiechert [30] which results in 7-queue layouts for general (that is, not necessarily planar) 3-trees. To reduce the number of required queues in the produced layouts, we make use of structural properties of the input graph. In particular, we put the main ideas of the algorithm of Wiechert [30] into a peeling-into-levels approach (see, e.g., [32]), according to which the vertices and the edges of the input graph are partitioned as follows:

- Vertices incident to the outerface are at level zero,

- Vertices incident to the outerface of the graph induced by deleting all vertices of levels $0, \ldots, i-1$ are at level $i$,

- Edges between same-level vertices are called level edges, and

- Edges between vertices of different levels are called binding edges. 
To keep the description simple, we first show how to compute a 5-queue layout of a planar 3-tree $G$, assuming that $G$ has only two levels (refer to Sect. 3.1). Then, we extend our approach to the general case of more than two levels (refer to Sect. 3.2). We conclude by discussing the differences between the approach of Wiechert [30] and ours (refer to Sect. 3.3); we also describe which properties of planar 3-trees we exploited to reduce the required number of queues.

\subsection{The Two-Level Case}

We start with the (intuitively easier) case in which the given planar 3-tree $G$ consists of two levels, which we denote by $L_{0}$ and $L_{1}$. Since we use this case as a tool to cope with the general case of more than two levels, we consider a slightly more general scenario. In particular, we make the following assumptions; see Fig. 3a for an illustration:

A. 1 the graph $G_{0}$ induced by the vertices of level $L_{0}$ is outerplane and internally triangulated, and

A. 2 each connected component of the graph $G_{1}$ induced by the vertices of level $L_{1}$ is outerplane and resides within a (triangular) face of graph $G_{0}$.

Without loss of generality we may also assume that graph $G_{0}$ is biconnected, as otherwise we can augment it to being biconnected by adding (level- $L_{0}$ ) edges without affecting its outerplanarity (see, e.g., the dotted-gray edges of Fig. 3a). Note that in a planar 3-tree, the graph induced by the vertices at level zero is simply a 3-cycle (and not any outerplane graph, as we have assumed), and as a result the graph induced by the vertices at level one is a single outerplane component. Our algorithm maintains the following invariants:

I.1 the linear order is such that all vertices of level $L_{0}$ precede all vertices of level $L_{1}$,

I.2 the level edges of $G$ are assigned to two queues, which we denote by $\mathcal{Q}_{0}$ and $\mathcal{Q}_{1}$, and

I.3 the binding edges between $L_{0}$ and $L_{1}$ of $G$ are assigned to three queues, which we denote by $\mathcal{Q}_{2}, \mathcal{Q}_{3}$, and $\mathcal{Q}_{4}$.

To compute an order that satisfies Invariant I.1, we construct two orders, one for the vertices of level $L_{0}$ (that satisfies Invariant I.2) and one for the vertices of level $L_{1}$ (that also satisfies Invariant I.2). Then, we concatenate them, so that all the vertices of level $L_{0}$ precede all the vertices of level $L_{1}$ (as required by Invariant I.1). Note that critical in this step is to also maintain Invariant I.3.

To compute an order of the vertices of level $L_{0}$ satisfying Invariant I.2, we directly apply Lemma 2, as by our initial assumption A.1, graph $G_{0}$ is internallytriangulated and outerplane. It follows that Invariant I.2 is satisfied for the vertices of level $L_{0}$. To compute an order of the vertices of level $L_{1}$ satisfying Invariant I.2, we apply Lemma 2 individually to each connected component of graph $G_{1}$, which by 


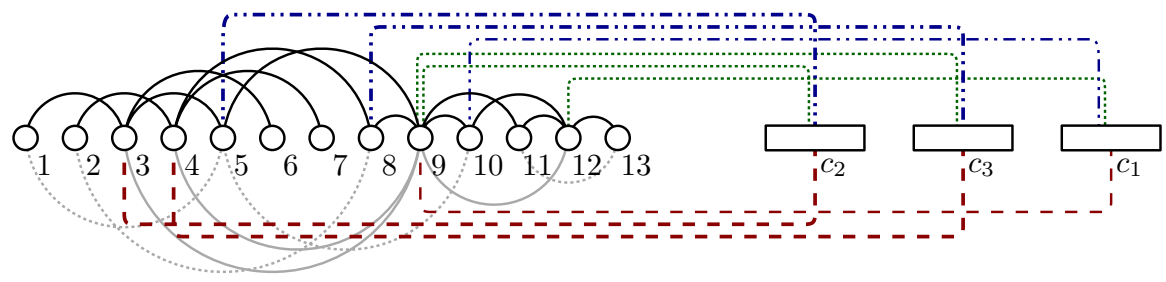

Fig. 4 The 5-queue layout for the graph in Fig. 3; since $5<8$ and $8<10$ in the order of the vertices of level $L_{0}$ as seen in Fig. 3, $c_{2}$ precedes $c_{3}$, and $c_{3}$ precedes $c_{1}$

our initial assumption A.2 is an internally-triangulated and outerplane graph. Then, the resulting orders are concatenated, such that for every two connected components of graph $G_{1}$, all vertices of the first one either precede or follow all vertices of the second one (critical at this point is to maintain Invariant I.3, as already mentioned above). The latter property allows us to use queues $\mathcal{Q}_{0}$ and $\mathcal{Q}_{1}$ for all the level edges of level $L_{1}$. Therefore, Invariant I.2 is satisfied.

To guarantee that Invariant I.3 is satisfied, we proceed as follows. Consider a connected component $c$ of $G_{1}$. By our initial assumption A.2, component $c$ resides within a triangular face $\langle u, v, w\rangle$ of graph $G_{0}$. Without loss of generality, let $u, v$ and $w$ be the anchor, top and bottom vertices of the face, respectively. We assign the binding edges incident to $u$ to queue $\mathcal{Q}_{2}$, the binding edges incident to $v$ to queue $\mathcal{Q}_{3}$ and the binding edges incident to $w$ to queue $\mathcal{Q}_{4}$; see the blue, red, and green edges in Fig. 4.

Next, we describe how to compute the relative order of the connected components of graph $G_{1}$. Let $c$ and $c^{\prime}$ be two such components. By our initial assumption A.2, components $c$ and $c^{\prime}$ reside within two triangular faces, say $\langle u, v, w\rangle$ and $\left\langle u^{\prime}, v^{\prime}, w^{\prime}\right\rangle$, of graph $G_{0}$. Without loss of generality, assume that $u$ and $u^{\prime}$ are the anchors, $v$ and $v^{\prime}$ are top, and $w$ and $w^{\prime}$ are bottom vertices of the two faces. If $u \neq u^{\prime}$, then component $c$ precedes component $c^{\prime}$ if and only if $u<u^{\prime}$ in the order of the vertices of level $L_{0}$. If $u=u^{\prime}$, we have $v \neq v^{\prime}$ or $w \neq w^{\prime}$. If $v \neq v^{\prime}$, then component $c$ precedes component $c^{\prime}$ if and only if $v<v^{\prime}$ in the order of the vertices of level $L_{0}$. Otherwise (that is, $u=u^{\prime}$ and $v=v^{\prime}$ ), component $c$ precedes component $c^{\prime}$ if and only if $w<w^{\prime}$ in the order of the vertices of level $L_{0}$. We claim that for the resulting order of the vertices of level $L_{1}$, Invariant I.3 is satisfied, that is, no two independent edges of each of the queues $\mathcal{Q}_{2}, \mathcal{Q}_{3}$ and $\mathcal{Q}_{4}$ are nested.

We start our proof with queue $\mathcal{Q}_{2}$. Consider two independent edges $(x, y) \in \mathcal{Q}_{2}$ and $\left(x^{\prime}, y^{\prime}\right) \in \mathcal{Q}_{2}$, where $x, x^{\prime} \in L_{0}$ and $y, y^{\prime} \in L_{1}$ (see the blue edges incident to vertices 5 and 8 in Fig. 4). By construction of queue $\mathcal{Q}_{2}$, vertices $x$ and $x^{\prime}$ are anchors of two distinct faces $f_{x}$ and $f_{x^{\prime}}$ of graph $G_{0}$ (see the faces of Fig. $3 \mathrm{~b}$ that contain components $c_{2}$ and $c_{3}$ ). Without loss of generality, we assume that $x<x^{\prime}$ in the order of the vertices of level $L_{0}$. It follows that the two components, say $c_{y}$ and $c_{y^{\prime}}$, of graph $G_{1}$, that reside within faces $f_{x}$ and $f_{x^{\prime}}$ and contain $y$ and $y^{\prime}$, respectively, are such that all vertices of component $c_{y}$ precede all vertices of component $c_{y^{\prime}}$ (in Fig. $4, x=5$ precedes $y=8$; thus, $c_{y}=c_{2}$ precedes $c_{y^{\prime}}=c_{3}$ ). Since $y \in c_{y}$ and $y^{\prime} \in c_{y^{\prime}}$, edges $(x, y)$ and $\left(x^{\prime}, y^{\prime}\right)$ are not nested, as desired. 
We continue our proof with queue $\mathcal{Q}_{3}$. Let $(x, y)$ and $\left(x^{\prime}, y^{\prime}\right)$ be two independent edges of $\mathcal{Q}_{3}$, where $x, x^{\prime} \in L_{0}$ and $y, y^{\prime} \in L_{1}$ (see the red edges in Fig. 4 incident to vertices 3 and 4). By construction of $\mathcal{Q}_{3}$, vertices $x$ and $x^{\prime}$ are the top vertices of two distinct faces $f_{x}$ and $f_{x^{\prime}}$ of graph $G_{0}$ (see the faces of Fig. $3 \mathrm{~b}$ that contain components $c_{2}$ and $c_{3}$ ). Let $c_{y}$ and $c_{y^{\prime}}$ be the components of graph $G_{1}$ that reside within faces $f_{x}$ and $f_{x^{\prime}}$ and contain vertices $y$ and $y^{\prime}$. Finally, let $u$ and $u^{\prime}$ be the anchors of faces $f_{x}$ and $f_{x^{\prime}}$, respectively. Suppose first that $u \neq u^{\prime}$ and assume that $u \prec u^{\prime}$ in the order the vertices of level $L_{0}$. Since $u \prec u^{\prime}$, by Property 1 it follows that $x \prec x^{\prime}$ and that all vertices of component $c_{y}$ precede all vertices of component $c_{y^{\prime}}$ (in Fig. 4, $u=5$ precedes $u^{\prime}=8$, which implies that $x=3$ precedes $x^{\prime}=4$; thus, $c_{y}=c_{2}$ precedes $c_{y}^{\prime}=c_{3}$ ). Since $y \in c_{y}$ and $y^{\prime} \in c_{y^{\prime}}$, it follows that edges $(x, y)$ and $\left(x^{\prime}, y^{\prime}\right)$ are not nested. Suppose now that $u=u^{\prime}$ and assume without loss of generality that $x<x^{\prime}$ in the order of the vertices of level $L_{0}$. Since $u=u^{\prime}$ and $x \prec x^{\prime}$, all vertices of component $c_{y}$ precede all vertices of component $c_{y^{\prime}}$. Since $y \in c_{y}$ and $y^{\prime} \in c_{y^{\prime}}$, it follows that edges $(x, y)$ and $\left(x^{\prime}, y^{\prime}\right)$ are not nested, as desired.

We conclude our proof with queue $\mathcal{Q}_{4}$. Let as above $(x, y)$ and $\left(x^{\prime}, y^{\prime}\right)$ be two independent edges of $\mathcal{Q}_{4}$, where $x, x^{\prime} \in L_{0}$ and $y, y^{\prime} \in L_{1}$. It follows that vertices $x$ and $x^{\prime}$ are the bottom vertices of two distinct faces $f_{x}$ and $f_{x^{\prime}}$ of graph $G_{0}$. Let $c_{y}$ and $c_{y^{\prime}}$ be the components of graph $G_{1}$ that reside within faces $f_{x}$ and $f_{x^{\prime}}$ and contain vertices $y$ and $y^{\prime}$. Finally, let $u$ and $u^{\prime}$ be the anchors, and $v$ and $v^{\prime}$ be the top vertices of faces $f_{x}$ and $f_{x^{\prime}}$, respectively. If $u \neq u^{\prime}$, or $u=u^{\prime}$ and $v \neq v^{\prime}$, then the proof that edges $(x, y)$ and $\left(x^{\prime}, y^{\prime}\right)$ are not nested follows using similar arguments as in corresponding cases of queue $\mathcal{Q}_{3}$. Thus, it remains to consider the case in which $u=u^{\prime}$ and $v=v^{\prime}$. Assume without loss of generality that $x \prec x^{\prime}$ in the order of the vertices of level $L_{0}$. It follows that all vertices of component $c_{y}$ precede all vertices of component $c_{y^{\prime}}$. Since $y \in c_{y}$ and $y^{\prime} \in c_{y^{\prime}}$, it follows that $(x, y)$ and $\left(x^{\prime}, y^{\prime}\right)$ are not nested. Hence, Invariant I.3 is satisfied, as desired.

The discussion above concludes the two-level case. However, before we proceed with the multi-level case, we make a useful observation. To satisfy Invariant I.3, we did not impose any restriction on the order of the vertices of each connected component of graph $G_{1}$ (any order that satisfies Invariant I. 2 for level $L_{1}$ would be suitable for us, that is, not necessarily the one constructed by Lemma 2). What we fixed, was the relative order of these components. With this observation in mind, we are now ready to proceed to the multi-level case.

\subsection{The Multi-Level Case}

We now consider the general case, in which our planar 3-tree $G$ consists of more than two levels, say $L_{0}, L_{1}, \ldots, L_{\lambda}$ with $\lambda \geq 2$. For $i=0,1, \ldots, \lambda$, let $G_{i}$ be the subgraph of $G$ induced by the vertices of level $L_{i}$. We claim that the connected components of each graph $G_{i}$ are internally-triangulated outerplane graphs that are not necessarily biconnected. Clearly, this holds for graph $G_{0}$, which is a 3-cycle. Assuming that, for some $i=1, \ldots, \lambda$, graph $G_{i-1}$ has the claimed property, we observe that each connected component of graph $G_{i}$ resides within a facial 3-cycle of graph $G_{i-1}$. Since each non-empty facial 3-cycle of graph $G_{i-1}$ induces a planar 3-tree in graph 
$G$ [21], the claim follows by observing that the removal of the outer face of a planar 3 -tree yields a plane graph, whose outer vertices induce an internally-triangulated outerplane graph.

For the recursive step of our algorithm, we will assume that for some $i \in\{0,1, \ldots, \lambda-1\}$ we have a 5 -queue layout for each of the connected components of the graph $H_{i+1}$ induced by the vertices of levels $L_{i+1}, \ldots, L_{\lambda}$, that satisfies the following invariants ${ }^{1}$ :

M.1 the linear order is such that all vertices of level $L_{j}$ precede all vertices of level $L_{j+1}$ for every $j=i+1, \ldots, \lambda-1$;

M.2 the level edges of each of the levels $L_{i+1}, \ldots, L_{\lambda}$ have been assigned to two queues, which we denote by $\mathcal{Q}_{0}$ and $\mathcal{Q}_{1}$;

M.3 for every $j=i+1, \ldots, \lambda-1$, the binding edges between $L_{j}$ and $L_{j+1}$ have been assigned to three queues, which we denote by $\mathcal{Q}_{2}, \mathcal{Q}_{3}$, and $\mathcal{Q}_{4}$.

In the following, we show how to construct a 5-queue layout (that satisfies Invariants M.1-M.3) for each of the connected components of the graph $H_{i}$ induced by the vertices of levels $L_{i}, \ldots, L_{\lambda}$. Let $C_{i}$ be such a component. By definition, $C_{i}$ is delimited by a connected component $c_{i}$ of graph $G_{i}$, which is internally-triangulated and outerplane. If none of the faces of component $c_{i}$ contains a connected component of $H_{i+1}$, then we compute a 2-queue layout of it using Lemma 2. Consider now the more general case, in which some of the faces of component $c_{i}$ contain connected components of $H_{i+1}$. By Invariants M.1-M.3, we may assume that we have computed 5-queue layouts for all the connected components, say $d_{1}, \ldots, d_{k}$, of $H_{i+1}$ that reside within the faces of component $c_{i}$.

We proceed by applying the two-level algorithm to the subgraph of $C_{i}$ induced by the vertices of $c_{i}$ and the vertices incident to the outer faces of $d_{1}, \ldots, d_{k}$. By the last observation that we made in the two-level case, this will result in:

i. A linear order $\mathcal{O}\left(c_{i}\right)$ of the vertices of component $c_{i}$,

ii. A relative order of the components $d_{1}, \ldots, d_{k}$,

iii. all vertices of $c_{i}$ precede those of $d_{1}, \ldots, d_{k}$,

iv. An assignment of the (level- $L_{i}$ ) edges of $c_{i}$ into $\mathcal{Q}_{0}$ and $\mathcal{Q}_{1}$, and

v. An assignment of the binding edges between $c_{i}$ and each of $d_{1}, \ldots, d_{k}$ into $\mathcal{Q}_{2}$, $\mathcal{Q}_{3}$ and $\mathcal{Q}_{4}$.

Up to renaming, we may assume without loss of generality that $d_{1}, \ldots, d_{k}$ is the computed order of these components; see Fig. 5a. By (iv) and (v), all edges of $C_{i}$ are assigned to $\mathcal{Q}_{0}, \ldots, \mathcal{Q}_{4}$, since the edges of components $d_{1}, \ldots, d_{k}$ have been recursively assigned to these queues. Next, we describe the order of the vertices of $C_{i}$. First, we partition the order of the vertices of $C_{i}$ into $\lambda-i+1$ disjoint intervals, say $I_{i}, \ldots, I_{\lambda}$, such that $I_{\mu}$ precedes $I_{\nu}$ if and only if $\mu<v$. All the (level- $L_{i}$ ) vertices of $c_{i}$

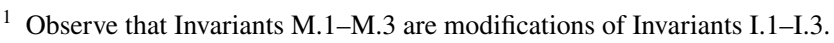




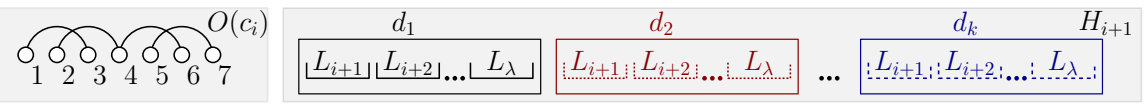

(a)

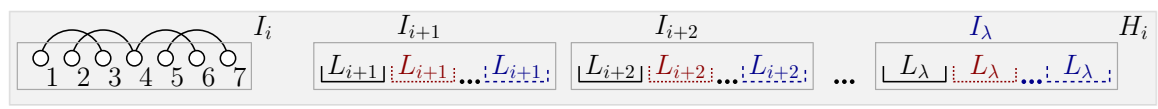

(b)

Fig. 5 Illustrations for the proof of Theorem 1. a For each of the components $d_{1}, \ldots, d_{k}$, all vertices of level $L_{j}$ precede all vertices of level $L_{j+1} ; j=i+1, \ldots, \lambda-1$, and $\mathbf{b}$ the computed linear order based on $p_{i}, \ldots, p_{\lambda}$

are contained in $I_{i}$ in the order $\mathcal{O}\left(c_{i}\right)$ by (i). For $j=i+1, \ldots, \lambda$, interval $I_{j}$ contains the vertices of level $L_{j}$ of each of the components $d_{1}, \ldots, d_{k}$, such that the vertices of level $L_{j}$ of component $d_{\mu}$ precede the vertices of level $L_{j}$ of component $d_{\nu}$ if and only if $\mu<v$; see Fig. 5b. We are not ready to state the main theorem of this section.

Theorem 1 Every planar 3-tree has queue number at most 5.

Proof Since Invariant M.1 is clearly satisfied for $C_{i}$, it remains to prove that the assignment of the edges of $C_{i}$ to queues $\mathcal{Q}_{0}, \ldots, \mathcal{Q}_{4}$ is such that Invariants M.2 and M.3 are satisfied.

Since the edges of $H_{i}$ are partitioned into level and binding, the endvertices of each edge are either in the same or in two consecutive intervals. In the former case, the edge is level and thus assigned either to $\mathcal{Q}_{0}$ or to $\mathcal{Q}_{1}$, while in the latter case the edge is binding and thus assigned to one of $\mathcal{Q}_{2}, \mathcal{Q}_{3}$ and $\mathcal{Q}_{4}$. Edges assigned to $\mathcal{Q}_{0}$ and $\mathcal{Q}_{1}$ cannot nest, as otherwise our two-level algorithm has computed an invalid assignment for the level edges of $c_{i}$ or an invalid assignment in $\mathcal{Q}_{0}$ and $\mathcal{Q}_{1}$ has been recursively computed for some of the components $d_{1} \ldots, d_{k}$. For the same reason, any two (binding) edges of $\mathcal{Q}_{2}, \mathcal{Q}_{3}$ or $\mathcal{Q}_{4}$ cannot be nested, if both bridge $c_{i}$ with the same or with two different components of level $L_{i+1}$, or both belong to the same component $d_{j}$, for some $j=1, \ldots, k$. It remains to prove that there exist no two nested edges of $\mathcal{Q}_{2}, \mathcal{Q}_{3}$ or $\mathcal{Q}_{4}$ that belong to two different components $d_{\mu}$ and $d_{\nu}$ and their endvertices are in two consecutive intervals $I_{j}$ and $I_{j+1}$, where $1 \leq \mu, v \leq k$ and $j=i, \ldots, \lambda-1$. This holds because all vertices of $d_{\mu}$ either precede or follow all vertices of $d_{v}$ in both $I_{j}$ and $I_{j+1}$ (by the choice of the relative order). Therefore, Invariants M.1-M.3 are satisfied and the proof follows.

\subsection{Differences with Wiechert's Algorithm}

We conclude this section by discussing the main differences between our algorithm and the previously best-known algorithm by Wiechert [30]. It is worth noting that the latter algorithm builds upon another algorithm by Dujmović et al. [10]. Both yield queue layouts for general (that is, not necessarily planar) $k$-trees, using the 
breadth-first search starting from an arbitrary vertex $r$ of graph $G$. For each $d>0$ and each connected component $C$ induced by the vertices at distance $d$ from vertex $r$, create a node (called bag) "containing" all vertices of component $C$; two bags are adjacent if and only if there is an edge of graph $G$ between them. For a $k$-tree, the result is a tree of bags, which we denote by $T$, called tree-partition, which has the following properties:

P.1. every node of $T$ induces a connected $(k-1)$-tree, and

P.2. for each non-root node $x \in T$, if $y \in T$ is the parent of $x$, then the vertices in $y$ having a neighbor in $x$ form a clique of size $k$.

Both algorithms order the bags of $T$ such that the vertices of the bags at distance $d$ from $r$ precede those at distance $d+1$. The vertices within each bag are ordered by induction using P.1. The algorithms differ in the way the edges are assigned to queues; the more efficient one by Wiechert [30] uses $2^{k}-1$ queues $\left(2^{k-1}\right.$ for the inter- and $2^{k-1}+1$ for the intra-bag edges), which is worst-case optimal for 1 - and 2-trees.

If graph $G$ is a planar 3-tree and the breadth-first search is started from a dummy vertex incident to the three outervertices of graph $G$, then the intra- and inter-bag edges correspond to the level and binding edges of our approach, while the bags at distance $d$ from $r$ in $T$ correspond to different connected components of level $d-1$.

To reduce the number of queues, we observed that in graph $G$ every node of treepartition $T$ induces a connected outerplanar graph, while each clique of size three by P.2 is a triangular face of graph $G$. By the first observation, we reduced the number of queues for intra-bag edges; by the second, we combined orders from different bags more efficiently.

\section{The Lower Bound}

In this section, we will prove that the queue number of planar 3-trees is at least four. To this end, we will define recursively a planar 3-tree and we will show that it contains at least one 4-rainbow in any linear order of its vertices. By Lemma 1, this directly implies that the queue number of this graph is at least four. Recall that according to the formal definition that we gave in Sect. 1, a planar 3-tree is a maximal planar graph. To keep the presentation simple, however, in this section we will relax this constraint by defining a subgraph of a planar 3-tree.

Starting with a set of $T$ independent edges $\left(s_{i}, t_{i}\right)$ with $1 \leq i \leq T$ and $T$ to be determined later, we connect their endpoints to two unique vertices, which we denote by $A$ and $B$; see Fig. 6a. We refer to these edges as $(s, t)$-edges. We also assume $A$ and $B$ to be adjacent. As a next step, we stellate each 3 -cycle $\left\langle A, s_{i}, t_{i}\right\rangle$ with a vertex $x_{i}$, that is, we introduce vertex $x_{i}$ and connect it to $A, s_{i}$ and $t_{i}$. Symmetrically, we also stellate each 3-cycle $\left\langle B, s_{i}, t_{i}\right\rangle$ with a vertex $y_{i}$. Afterwards, we perform a second round of stellations, where we stellate each of the 3-cycles $\left\langle x_{i}, s_{i}, t_{i}\right\rangle,\left\langle y_{i}, s_{i}, t_{i}\right\rangle,\left\langle A, x_{i}, s_{i}\right\rangle$, $\left\langle A, x_{i}, t_{i}\right\rangle,\left\langle B, y_{i}, s_{i}\right\rangle$ and $\left\langle B, y_{i}, t_{i}\right\rangle$ with vertices $\alpha_{i}, \beta_{i}, p_{i}, q_{i}, u_{i}$ and $v_{i}$, respectively. We 


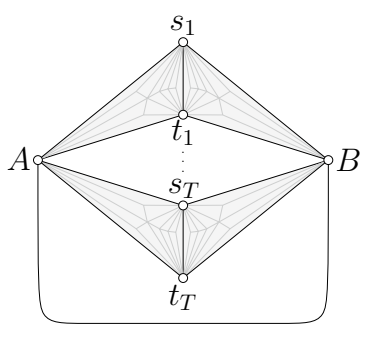

(a)

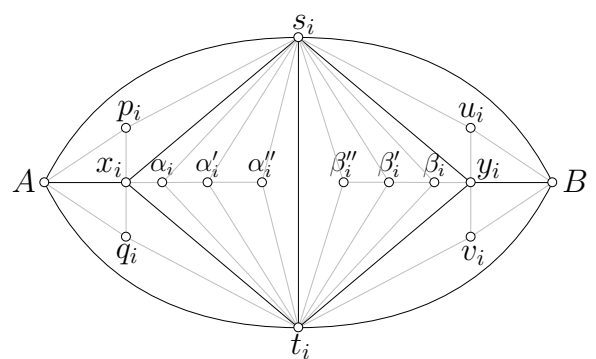

(b)

Fig. 6 Construction of graph $G_{T}$ : Each gray subgraph in a corresponds to a copy of the graph of $\mathbf{b}$

further stellate $\left\langle s_{i}, t_{i}, \alpha_{i}\right\rangle$ with $\alpha_{i}^{\prime}$ and then $\left\langle s_{i}, t_{i}, \alpha_{i}^{\prime}\right\rangle$ with $\alpha_{i}^{\prime \prime}$. Symmetrically, we stellate $\left\langle s_{i}, t_{i}, \beta_{i}\right\rangle$ with $\beta_{i}^{\prime}$, and $\left\langle s_{i}, t_{i}, \beta_{i}^{\prime}\right\rangle$ with $\beta_{i}^{\prime \prime}$. For an illustration refer to Fig. $6 \mathrm{~b}$.

Let $G_{T}$ be the graph constructed so far. We refer to vertices $A$ and $B$ as the poles of $G_{T}$ and we assume that $G_{T}$ admits a 3-queue layout $\mathcal{Q}$. By symmetry, we may assume without loss of generality that $A \prec B$ and that $s_{i} \prec t_{i}$ for each edge $\left(s_{i}, t_{i}\right)$. Consider a single edge $\left(s_{i}, t_{i}\right)$ and the relative order of its endvertices with respect to the poles $A$ and $B$ of graph $G_{T}$. Clearly, there exist six possible permutations:

P.1. $s_{i} \prec A \prec B \prec t_{i}$,

P.2. $A \prec s_{i}<B<t_{i}$,

P.3. $s_{i} \prec A \prec t_{i} \prec B$,

P.4. $A \prec B<s_{i}<t_{i}$,

P.5. $s_{i}<t_{i}<A<B$, and

P.6. $A \prec s_{i} \prec t_{i} \prec B$.

The main steps in our proof are the following. We first observe that by the pigeonhole principle and by setting $T=6 \tau$, at least one of the permutations P.1, ., P.6 applies to at least $\tau$ edges. Then, we show that if "too many" $(s, t)$-edges share one of the permutations P.1, ., P.5, there exists a 4-rainbow regardless of the linear order of the vertices of $G_{T}$, which by Lemma 1 contradicts the fact that $\mathcal{Q}$ is a 3-queue layout for graph $G_{T}$. Therefore, if $T$ is large enough, then for at least one $(s, t)$-edge of $G_{T}$ permutation P.6 applies. Based on this implication, we describe how to augment graph $G_{T}$, such that we can also rule out permutation P.6. Thereby, proving the claimed lower bound of four. We start with an auxiliary lemma.

Lemma 3 In every queue that contains $r^{2}$ independent edges, there exists either an $r$-twist or an r-necklace.

Proof Assume that no $r$-twist exists, as otherwise there is nothing to prove. We will prove the existence of an $r$-necklace. Let $\left(s_{1}, t_{1}\right), \ldots,\left(s_{r^{2}}, t_{r^{2}}\right)$ be the $r^{2}$ independent edges such that $s_{i} \prec t_{i}$, for $i=1, \ldots, r^{2}$. Assume without loss of generality that $s_{i} \prec s_{i+1}$ for each $i=1, \ldots, r^{2}-1$. Consider the edge $\left(s_{1}, t_{1}\right)$. Since $s_{1}$ is the first vertex in the order and no two edges nest, each vertex $t_{i}$, with $i>1$, is to the 


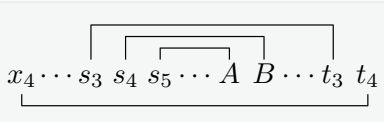

(a) $x_{4} \prec s_{3}$

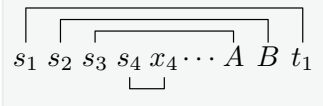

(b) $s_{3} \prec x_{4} \prec A$

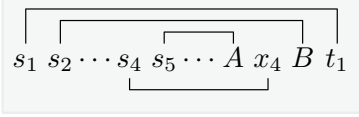

(c) $A \prec x_{4} \prec B$

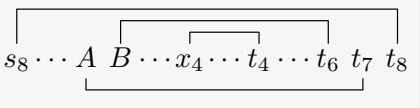

(d) $B \prec x_{4} \prec t_{6}$

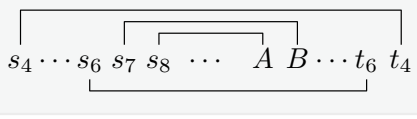

(e) $t_{6} \prec x_{4}$

Fig. 7 Illustration for the Case P.1

right of $t_{1}$. Since no $r$-twist exists, vertex $s_{r}$ is to the right of vertex $t_{1}$. Thus, edges $\left(s_{1}, t_{1}\right)$ and $\left(s_{r}, t_{r}\right)$ do not cross. The removal of the edges $\left(s_{1}, t_{1}\right), \ldots,\left(s_{r-1}, t_{r-1}\right)$ makes vertex $s_{r}$ first. By applying this argument $r-1$ times, we conclude that $\left(s_{1}, t_{1}\right),\left(s_{r}, t_{r}\right), \ldots\left(s_{(r-1)^{2}+1}, t_{(r-1)^{2}+1}\right)$ form an $r$-necklace and the proof of the lemma follows.

Applying the pigeonhole principle to a $k$-queue layout, we obtain the following.

Corollary 1 Every $k$-queue layout with at least $k r^{2}$ independent edges contains at least one r-twist or at least one $r$-xnecklace.

We exploit Corollary 1 as follows. Recall that $\mathcal{Q}$ is a 3-queue layout for graph $G_{T}$. So, if we set $T=18 r^{2}$ for an $r>0$ of our choice, then at least $3 r^{2}(s, t)$-edges of graph $G_{T}$ share the same permutation. Since these edges are by construction independent, by Corollary 1 at least $r$ of them, say without loss of generality $\left(s_{1}, t_{1}\right), \ldots,\left(s_{r}, t_{r}\right)$, form a necklace or a twist (while also sharing the same permutation). In the following, we show that, for an appropriate choice of $r$, if $\left(s_{1}, t_{1}\right), \ldots,\left(s_{r}, t_{r}\right)$ form a necklace or a twist and simultaneously share one of the permutations P. $1, \ldots$, P.5, then a 4-rainbow is inevitably induced, which by Lemma 1 contradicts the fact that $\mathcal{Q}$ is a 3-queue layout for $G_{T}$. We consider each case separately.

Case P.1 Let $r=8$. It is sufficient to consider the case in which the edges $\left(s_{1}, t_{1}\right), \ldots,\left(s_{8}, t_{8}\right)$ form a twist, since for $r>1$ the necklace case is impossible. Since $\left(s_{1}, t_{1}\right), \ldots,\left(s_{8}, t_{8}\right)$ share permutation P.1, the order is $\left[s_{1} \ldots s_{8} A B t_{1} \ldots t_{8}\right]$. In the following, we prove the existence of a 4-rainbow, contradicting the fact that $\mathcal{Q}$ is a 3-queue layout for graph $G_{T}$. More precisely:

- if $x_{4}<s_{3}$, then the edges $\left(x_{4}, t_{4}\right),\left(s_{3}, t_{3}\right),\left(s_{4}, B\right)$ and $\left(s_{5}, A\right)$ form a 4-rainbow; see Fig. $7 \mathrm{a}$,

- if $s_{3} \prec x_{4} \prec A$, then the edges $\left(s_{1}, t_{1}\right),\left(s_{2}, B\right),\left(s_{3}, A\right)$ and $\left(s_{4}, x_{4}\right)$ form a 4-rainbow; see Fig. 7b,

- if $A<x_{4}<B$, then the edges $\left(s_{1}, t_{1}\right),\left(s_{2}, B\right),\left(s_{4}, x_{4}\right)$ and $\left(s_{5}, A\right)$ form a 4-rainbow; see Fig. 7c, 


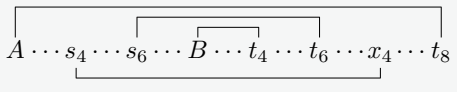

(a) $t_{6} \prec x_{4} \prec t_{8}$

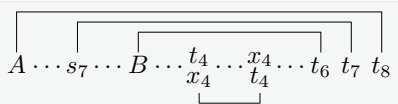

(b) $B \prec x_{4} \prec t_{6}$

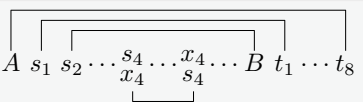

(c) $s_{3} \prec x_{4} \prec B$

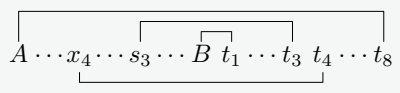

(d) $A \prec x_{4} \prec s_{3}$

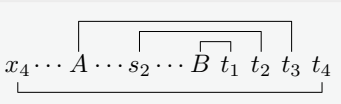

(e) $x_{4} \prec A$

Fig. 8 Illustration for the Case P.2 when $x_{4} \prec t_{8}$ holds

- if $B \prec x_{4} \prec t_{6}$, then the edges $\left(s_{8}, t_{8}\right),\left(A, t_{7}\right),\left(B, t_{6}\right)$ and $\left(x_{4}, t_{4}\right)$ form a 4-rainbow; see Fig. 7d, and

- if $t_{6} \prec x_{4}$, then the edges $\left(s_{4}, t_{4}\right),\left(s_{6}, t_{6}\right),\left(s_{7}, B\right)$ and $\left(s_{8}, A\right)$ form a 4-rainbow; see Fig. 7e.

Since each subcase yields a 4-rainbow, the proof of Case P.1 follows.

Case P.2 As in the previous case, we set $r=8$ and we only consider the case, in which $\left(s_{1}, t_{1}\right), \ldots,\left(s_{8}, t_{8}\right)$ form a twist, since the necklace case is again impossible. Hence, the order is $\left[A s_{1} \ldots s_{8} B t_{1} \ldots t_{8}\right]$. First, we claim that $t_{8} \prec x_{4}$. To prove the claim, assume to the contrary that $x_{4} \prec t_{8}$. In the following, we prove that this assumption inevitably implies a 4-rainbow, contradicting the fact that $\mathcal{Q}$ is a 3 -queue layout for graph $G_{T}$. More precisely:

- if $t_{6} \prec x_{4} \prec t_{8}$, then the edges $\left(A, t_{8}\right),\left(s_{4}, x_{4}\right),\left(s_{6}, t_{6}\right)$ and $\left(B, t_{4}\right)$ form a 4-rainbow; see Fig. $8 \mathrm{a}$,

- if $B \prec x_{4} \prec t_{6}$, then the edges $\left(A, t_{8}\right),\left(s_{7}, t_{7}\right),\left(B, t_{6}\right)$ and $\left(x_{4}, t_{4}\right)$ form a 4-rainbow; see Fig. 8b,

- if $s_{3} \prec x_{4} \prec B$, then the edges $\left(A, t_{8}\right),\left(s_{1}, t_{1}\right),\left(s_{2}, B\right)$ and $\left(s_{4}, t_{4}\right)$ form a 4-rainbow; see Fig. 8c,

- if $A \prec x_{4} \prec s_{3}$, then the edges $\left(A, t_{8}\right),\left(x_{4}, t_{4}\right),\left(s_{3}, t_{3}\right)$ and $\left(B, t_{1}\right)$ form a 4-rainbow; see Fig. $8 \mathrm{~d}$, and

- if $x_{4} \prec A$, then the edges $\left(x_{4}, t_{4}\right),\left(A, t_{3}\right),\left(s_{2}, t_{2}\right)$ and $\left(B, t_{1}\right)$ form a 4-rainbow; see Fig. 8e.

An analogous case distinction yields that $t_{8} \prec x_{5}$ must also hold. However, we have no knowledge about the relative order of $x_{4}$ and $x_{5}$. In the following, we show that regardless of the relative order of $x_{4}$ and $x_{5}$, a 4-rainbow is inevitably formed. More precisely:

- if $x_{4} \prec x_{5}$, then the edges $\left(A, x_{5}\right),\left(s_{4}, x_{4}\right),\left(s_{8}, t_{8}\right)$ and $\left(B, t_{4}\right)$ form a 4-rainbow; see Fig. 9a, and 


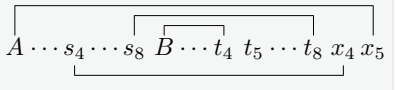

(a) $t_{8} \prec x_{4} \prec x_{5}$

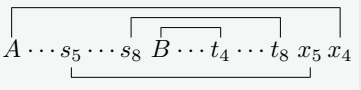

(b) $t_{8} \prec x_{5} \prec x_{4}$

Fig. 9 Illustration for the Case P. 2 when $t_{8} \prec x_{4}$ and $t_{8} \prec x_{5}$ hold

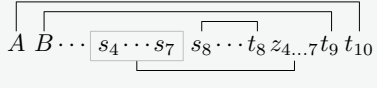

(a) $t_{8} \prec z_{4 \ldots 7} \prec t_{9}$

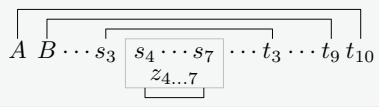

(c) $s_{3} \prec z_{4} \ldots 7 \prec t_{3}$

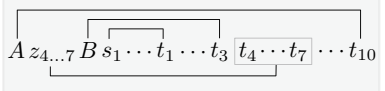

(e) $A \prec z_{4 \ldots 7} \prec B$

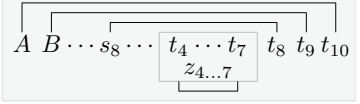

(b) $t_{3} \prec z_{4} \ldots 7 \prec t_{8}$

(d) $B \prec z_{4} \ldots 7 \prec s_{3}$

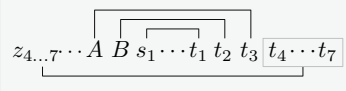

(f) $z_{4 \ldots 7} \prec A$

Fig. 10 Illustration for the Case P.4 when $z_{4 \ldots 7} \prec t_{9}$ holds

- if $x_{5} \prec x_{4}$, then the edges $\left(A, x_{4}\right),\left(s_{5}, t_{5}\right),\left(s_{8}, t_{8}\right)$ and $\left(B, t_{4}\right)$ form a 4-rainbow; see Fig. 9b.

Since each subcase yields a 4-rainbow, the proof of this case follows.

Case P.3 This case can be ruled out as Case P.2 due to symmetry.

Case P.4 We set $r=10$ and we proceed by distinguishing two subcases based on whether the edges $\left(s_{1}, t_{1}\right), \ldots,\left(s_{10}, t_{10}\right)$ form a twist or a necklace. Note that in contrast to the previous cases, here both subcases are possible.

We start with the twist case. Hence, the order is $\left[A B s_{1} \ldots s_{10} t_{1} \ldots t_{10}\right]$. Let $Z_{4 \ldots 7}=\left\{x_{4}, \ldots, x_{7}\right\} \cup\left\{y_{4}, \ldots y_{7}\right\}$ and let $z_{4 \ldots 7}$ be any element of $Z_{4 \ldots 7}$. By construction of graph $G_{T}$, vertex $z_{4 \ldots 7}$ has a neighbor in $\left\{s_{4}, \ldots, s_{7}\right\}$, and a neighbor in $\left\{t_{4}, \ldots, t_{7}\right\}$. Denote by $s_{4 \ldots 7}$ the endvertex of the former neighbor. Symmetrically, denote by $t_{4 \ldots 7}$ the latter neighbor. First, we claim that $t_{9} \prec z_{4} \ldots 7$, that is, all $x_{4}, \ldots, x_{7}$ and $y_{4}, \ldots, y_{7}$ are preceded by $t_{9}$. To prove the claim, assume to the contrary that $z_{4 \ldots 7} \prec t_{9}$. In the following, we prove that this assumption inevitably implies a 4-rainbow, contradicting the fact that $\mathcal{Q}$ is a 3 -queue layout for graph $G_{T}$. More precisely:

- if $t_{8} \prec z_{4 \ldots 7} \prec t_{9}$, then the edges $\left(A, t_{10}\right),\left(B, t_{9}\right),\left(s_{4 \ldots 7}, z_{4} \ldots 7\right)$ and $\left(s_{8}, t_{8}\right)$ form a 4-rainbow; see Fig. 10a, 
- if $t_{3} \prec z_{4 \ldots 7} \prec t_{8}$, then the edges $\left(A, t_{10}\right),\left(B, t_{9}\right),\left(s_{8}, t_{8}\right)$ and $\left(z_{4 \ldots 7}, t_{4 \ldots 7}\right)$ form a 4-rainbow; see Fig. 10b,

- if $s_{3} \prec z_{4 \ldots 7} \prec t_{3}$, then the edges $\left(A, t_{10}\right),\left(B, t_{9}\right),\left(s_{3}, t_{3}\right)$ and $\left(s_{4} \ldots 7, z_{4} \ldots 7\right)$ form a 4-rainbow; see Fig. 10c,

- if $B \prec z_{4 \ldots 7} \prec s_{3}$, then the edges $\left(A, t_{10}\right),\left(B, t_{9}\right),\left(z_{4 . \ldots 7}, t_{4 \ldots 7}\right)$ and $\left(s_{3}, t_{3}\right)$ form a 4-rainbow; see Fig. 10d,

- if $A \prec z_{4} \ldots 7<B$, then the edges $\left(A, t_{10}\right),\left(z_{4 \ldots 7}, t_{4 \ldots 7}\right),\left(B, t_{3}\right)$ and $\left(s_{1}, t_{1}\right)$ form a 4-rainbow; see Fig. 10e, and

- if $z_{4 \ldots 7} \prec A$, then the edges $\left(z_{4 \ldots 7}, t_{4 \ldots 7}\right),\left(A, t_{3}\right),\left(B, t_{2}\right)$ and $\left(s_{1}, t_{1}\right)$ form a 4-rainbow; see Fig. 10f.

From the above case analysis, it follows that $t_{9} \prec z_{4} \ldots 7$, that is, all $x_{4}, \ldots, x_{7}$ and $y_{4}, \ldots, y_{7}$ are preceded by $t_{9}$. Let us now take a closer look at the ordering of the eight vertices in $Z_{4 \ldots 7}$. We claim that their ordering has to comply with the following two requirements:

R.1. the indices of the first 7 elements of $Z_{4} \ldots 7$ are non-decreasing, and

R.2. for the last 7 elements of $Z_{4 . . .7}$, all $x_{i}$ 's precede all $y_{j}$ 's.

Assume to the contrary, that R.1 does not hold. Then, there exists a pair of vertices, say without loss of generality $x_{i}$ and $x_{j}$ with $i<j$, such that $x_{j} \prec x_{i}$, and $x_{i}$ is not the last element of $Z_{4 \ldots 7}$. Since the last element of $Z_{4 \ldots 7}$ has a connection to either $A$ or $B$, this connection and the edges $\left(s_{i}, x_{i}\right),\left(s_{j}, x_{j}\right)$ and $\left(s_{9}, t_{9}\right)$ form a 4-rainbow, contradicting the fact that $\mathcal{Q}$ is a 3-queue layout for graph $G_{T}$. Hence, R.1 holds, as desired.

To complete the proof of our claim, now assume to the contrary that R. 2 does not hold. Then, there exists a pair of vertices, say without loss of generality $x_{i}$ and $y_{j}$, such that $y_{j} \prec x_{i}$, and $y_{j}$ is not the first element of $Z_{4 \ldots 7}$. Without loss of generality, let $x_{k}$ be the first element of $Z_{4 \ldots 7}$. Then, the edges $\left(A, x_{i}\right),\left(B, y_{j}\right),\left(s_{k}, x_{k}\right)$ and $\left(s_{9}, t_{9}\right)$ form a 4 -rainbow, contradicting the fact that $\mathcal{Q}$ is a 3 -queue layout for graph $G_{T}$. Hence, R.2 holds, as desired.

Now, we show that R.1 and R.2 cannot simultaneously hold, which by our claim implies the existence of a 4-rainbow. Consider the last element of $Z_{4 \ldots 7}$. Assume that R.1 and R.2 both hold. By R.2, we may deduce that the last three elements of $Z_{4 \ldots 7}$ belong to $\left\{y_{4}, \ldots y_{7}\right\}$. Let them be $y_{i}, y_{j}, y_{\ell}$ as they appear from left to right. Then, by R.1 we have that $i<j$. Consider now $x_{j}$. By R. $1, y_{i} \prec x_{j}$ must hold. This contradicts the fact that $y_{i}, y_{j}, y_{\ell}$ are the last three elements of $Z_{4 . . .7}$.

We continue with the necklace case. Here, the order is $\left[A B s_{1} t_{1} \ldots s_{10} t_{10}\right]$. In the following, we make several observations in the form of propositions.

Proposition 1 Let $w_{i}$ be a neighbor of $s_{i}$ or $t_{i}$ not in $\left\{A, B, s_{i}, t_{i}\right\}$, for $3 \leq i \leq 8$. Then, either $s_{i-1} \prec w_{i} \prec t_{i+1}$, or $s_{10} \prec w_{i}$ holds in $\mathcal{Q}$. 


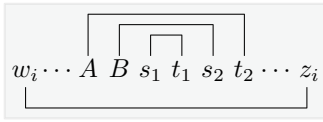

(a) $w \prec A$

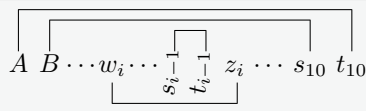

(c) $B \prec w \prec s_{i-1}$

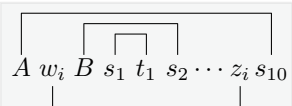

(b) $A \prec w \prec B$

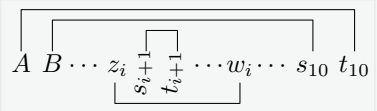

(d) $t_{i+1} \prec w \prec s_{10}$

Fig. 11 Illustrations for the proof of Proposition 1

Proof Let $z_{i} \in\left\{s_{i}, t_{i}\right\}$ be the neighbor of vertex $w_{i}$. We prove in the following that for any placement of $w_{i}$ such that neither $s_{i-1} \prec w_{i} \prec t_{i+1}$ nor $s_{10} \prec w_{i}$ hold, there is a 4-rainbow:

- if $w_{i} \prec A$, then the edges $\left(w_{i}, z_{i}\right),\left(A, t_{2}\right),\left(B, s_{2}\right)$ and $\left(s_{1}, t_{1}\right)$ form a 4-rainbow; see Fig. 11a;

- if $A \prec w_{i} \prec B$, then the edges $\left(A, s_{10}\right),\left(w_{i}, z_{i}\right),\left(B, s_{2}\right)$ and $\left(s_{1}, t_{1}\right)$ form a 4-rainbow; see Fig. 11b;

- if $B \prec w_{i} \prec s_{i-1}$, then $\left(A, t_{10}\right),\left(B, s_{10}\right),\left(w_{i}, z_{i}\right)$ and $\left(s_{i-1}, t_{i-1}\right)$ form a 4-rainbow; see Fig. 11c, and

- if $t_{i+1} \prec w_{i} \prec s_{10}$, then $\left(A, t_{10}\right),\left(B, s_{10}\right),\left(z_{i}, w_{i}\right)$ and $\left(s_{i+1}, t_{i+1}\right)$ form a 4-rainbow; see Fig. 11d.

Since each case yields a 4-rainbow, the proof follows.

Proposition 2 Let $w_{i}$ and $z_{i}$ be two vertices not in $\{A, B\}$ that form a $K_{4}$ with $s_{i}$ and $t_{i}$ for $3 \leq i \leq 8$. Then, at least one of the following holds in $\mathcal{Q}: s_{10} \prec w_{i}$ or $s_{10} \prec z_{i}$.

Proof The edges of the $K_{4}$ formed by the vertices $s_{i}, t_{i}, w_{i}$ and $z_{i}$ induce a 2-rainbow regardless of the relative order of their endvertices. By Proposition 1, each of $w_{i}$ and $z_{i}$ is either between $s_{i-1}$ and $t_{i+1}$, or after $s_{10}$. But if both $w_{i}$ and $z_{i}$ were between $s_{i-1}$ and $t_{i+1}$, then the 2-rainbow composed of the edges of the $K_{4}$, along with the two edges $\left(A, t_{10}\right),\left(B, s_{10}\right)$, would form a 4-rainbow; a contradiction. Hence, $s_{10} \prec w_{i}$ or $s_{10} \prec z_{i}$ must hold, as desired.

Proposition 3 For $4 \leq i \leq 8$, each vertex from the set $\left\{x_{i}, y_{i}, p_{i}, q_{i}, u_{i}, v_{i}\right\}$ is between $s_{i-1}$ and $t_{i+1}$ in $\mathcal{Q}$.

Proof Let $w_{i}$ be an arbitrary vertex from the set $\left\{x_{i}, y_{i}, p_{i}, q_{i}, u_{i}, v_{i}\right\}$. Observe that, by construction of graph $G_{T}$, vertex $w_{i}$ has an edge (which we call long) to exactly one of $A$ and $B$, and an edge (which we call short) to at least one of $s_{i}$ and $t_{i}$. By Proposition 1 , it is sufficient to prove that $w_{i}$ is not after $s_{10}$. Assume for a contradiction that $s_{10} \prec w_{i}$. 


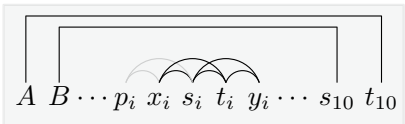

(a) $x_{i} \prec s_{i} \prec t_{i} \prec y_{i}$

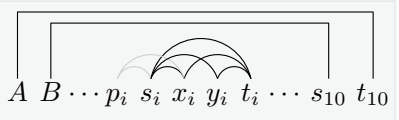

(b) $s_{i} \prec x_{i} \prec y_{i} \prec t_{i}$

Fig. 12 Contradiction for placing $x_{i}, y_{i}, p_{i}, q_{i}, u_{i}, v_{i}$ in range $\left(s_{i-1}, t_{i+1}\right), 4 \leq i \leq 8$

Consider the edges $\left(x_{i-1}, \alpha_{i-1}\right),\left(\alpha_{i-1}^{\prime}, \alpha_{i-1}^{\prime \prime}\right),\left(y_{i-1}, \beta_{i-1}\right),\left(\beta_{i-1}^{\prime}, \beta_{i-1}^{\prime \prime}\right)$, and observe that the endpoints of each of these edges create a $K_{4}$ with $s_{i-1}$ and $t_{i-1}$. By Proposition 2 , one endvertex from each of these four edges is after $s_{10}$. By the pigeonhole principle, at least two of these endvertices either precede or follow $w_{i}$. Call them $a_{i-1}$ and $b_{i-1}$, where without loss of generality $a_{i-1} \prec b_{i-1}$. Then, the edges $\left(s_{i-1}, b_{i-1}\right),\left(t_{i-1}, a_{i-1}\right)$ and $\left(s_{9}, t_{9}\right)$ form a 3-rainbow, since $s_{i-1} \prec t_{i-1} \prec s_{9} \prec t_{9} \prec s_{10} \prec a_{i-1} \prec b_{i-1}$. This 3-rainbow together with the short edge of $w_{i}$ (when both $a_{i-1}$ and $b_{i-1}$ follow $w_{i}$, that is, $w_{i} \prec a_{i-1} \prec b_{i-1}$ ), or with the long edge of $w_{i}$ (when both $a_{i-1}$ and $b_{i-1}$ precede $w_{i}$, that is, $a_{i-1} \prec b_{i-1} \prec w_{i}$ ) yield a 4-rainbow; a contradiction.

Since $s_{i}$ and $t_{i}$ are between $s_{i-1}$ and $t_{i+1}$, it follows from Proposition 3, that each vertex from the set $\left\{s_{i}, t_{i}, x_{i}, y_{i}, p_{i}, q_{i}, u_{i}, v_{i}\right\}$ is between $s_{i-1}$ and $t_{i+1}$, for $4 \leq i \leq 8$. Then, the edges between these vertices cannot form a 2-rainbow, as otherwise this 2-rainbow along with the two edges $\left(A, t_{10}\right)$ and $\left(B, s_{10}\right)$ would form a 4-rainbow, contradicting the fact that $\mathcal{Q}$ is a 3 -queue layout for $G_{T}$. Assume without loss of generality that $x_{i} \prec y_{i}$. Since $\left(x_{i}, y_{i}\right)$ is the only missing edge between vertices $\left\{x_{i}, y_{i}, s_{i}, t_{i}\right\}$, it follows that in order to avoid a 2-rainbow, we may assume that one of the following two cases applies (for an illustration, refer to Fig. 12):

- $x_{i} \prec s_{i} \prec t_{i} \prec y_{i}$,

$-s_{i} \prec x_{i} \prec y_{i} \prec t_{i}$.

In both cases, vertex $p_{i}$ must precede both $x_{i}$ and $s_{i}$, as otherwise either $\left(p_{i}, s_{i}\right),\left(x_{i}, t_{i}\right)$, or $\left(p_{i}, x_{i}\right),\left(s_{i}, t_{i}\right)$ would form a 2-rainbow; see Fig. 12. But then there is no valid position for $q_{i}$ without creating a 2-rainbow in either case, resulting together with $\left(A, t_{10}\right)$ and $\left(B, s_{10}\right)$ in a 4-rainbow. This completes the description of Case P.4.

Case P.5 This case can be ruled out as Case P.4 due to symmetry.

From the above case analysis it follows that if $r$ is at least 10 (which implies that $T$ is at least 1,800), then for at least one $(s, t)$-edge of $G_{T}$ permutation P.6 applies, that is, there exists $1 \leq i_{0} \leq T$ such that $A \prec s_{i_{0}} \prec t_{i_{0}} \prec B$. Notice that the edges $(A, B)$ and $\left(s_{i_{0}}, t_{i_{0}}\right)$ form a 2-rainbow.

We proceed by augmenting graph $G_{T}$ as follows. For each edge $\left(s_{i}, t_{i}\right)$ of graph $G_{T}$, we introduce a new copy of graph $G_{T}$, which has $s_{i}$ and $t_{i}$ as poles. Let $G_{T}^{\prime}$ be the augmented graph and let $\left(s_{1}^{\prime}, t_{1}^{\prime}\right), \ldots,\left(s_{T}^{\prime}, t_{T}^{\prime}\right)$ be the $(s, t)$-edges of the copy of graph $G_{T}$ in $G_{T}^{\prime}$ corresponding to the edge $\left(s_{i_{0}}, t_{i_{0}}\right)$ of the original graph $G_{T}$. Then, by our arguments above, in any 3-queue layout of $G_{T}^{\prime}$, there exists $1 \leq i_{1} \leq T$, such that 
$s_{i_{0}} \prec s_{i_{1}}^{\prime} \prec t_{i_{1}}^{\prime} \prec t_{i_{0}}$. Hence, the edges $(A, B),\left(s_{i_{0}}, t_{i_{0}}\right)$ and $\left(s_{i_{1}}^{\prime}, t_{i_{1}}^{\prime}\right)$ form a 3-rainbow, since $A \prec s_{i_{0}} \prec t_{i_{0}} \prec B$ holds. If we apply the same augmentation procedure to graph $G_{T}^{\prime}$, then we guarantee that the resulting graph $G_{T}^{\prime \prime}$, which is clearly a subgraph of a planar 3-tree, has inevitably a 4-rainbow. Hence, Theorem 2 follows. We stress here that graph $G_{T}^{\prime \prime}$ is rather large. Since $T \geq 1,800$, the total number of $(s, t)$-edges in $G_{T}^{\prime \prime}$ is at least $1,800^{3}$.

Theorem 2 There exist planar 3-trees that have queue number at least 4.

\section{Track Layouts}

In this section, we discuss implications of our results to the closely-related track layouts [11], which are formally defined as follows. Let $\left\{V_{i}: 1 \leq i \leq t\right\}$ be a partition of the vertex set of a graph $G$ such that for every edge $(u, v)$ of $G$, if $u \in V_{i}$ and $v \in V_{j}$, then $i \neq j$. Suppose that $<_{i}$ is a total order of $V_{i}$. Then, the ordered $\operatorname{set}\left(V_{i},<_{i}\right)$ is called a track and the partition is called a $t$-track assignment of $G$. An $X$-crossing in a track assignment consists of two edges $(u, v)$ and $\left(u^{\prime}, v^{\prime}\right)$ such that $u$ and $u^{\prime}$ are on the same track $V_{i}, v$ and $v^{\prime}$ are on a different track $V_{j}$ with $u<_{i} u^{\prime}$ and $v^{\prime}<_{j} v$. A track layout is a track assignment with no X-crossings. In other words, a track layout of a graph is a partition of its vertices into a number of tracks, such that the vertices in each track form an independent set and the edges between each pair of tracks form a non-crossing set. The track number of graph $G$ is the minimum $t$, such that $G$ has a $t$-track layout.

Track and queue layouts are closely related to each other, as observed by Dujmović et al. [11]. In particular, every graph that admits a $t$-track layout has queue number at most $(t-1)$, while every graph that admits a $q$-queue layout has track number at most $4 q \cdot 4^{q(2 q-1)(4 q-1)}$. For the case of graphs with bounded tree-width, several upper bounds on the track number are known; trees have track number 3 [14], outerplanar graphs have track number 5 [11], series-parallel graphs have track number at most 15 [6], and planar 3-trees have track number at most 5415 [6].

In the following, we improve the upper bound on the track number of planar 3 -trees, utilizing the following relation between the acyclic chromatic number of a graph admitting a $q$-queue layout and its track number. Recall that a vertex coloring is acyclic, if there is no bichromatic cycle, that is, all cycles consists of at least three colors.

Lemma 4 (Dujmović et al. [10]) Every q-queue graph with acyclic chromatic number $c$ has track-number at most $c(2 q)^{c-1}$.

Since the unique 4-coloring of a planar 3-tree is acyclic [15], combining Lemma 4 with Theorem 1 we obtain the following result.

Theorem 3 The track number of a planar 3-tree is at most 4000. 
Notice that the upper bound of Theorem 3 has been recently improved [26].

\section{Conclusions}

In this work, we presented improved bounds on the queue number of planar 3-trees. We conclude by mentioning two interesting open problems that arise from our work:

- The first one concerns the exact upper bound on the queue number of planar 3-trees. Does there exist a planar 3-tree whose queue number is five (as our upper bound) or the queue number of every planar 3-tree is four (as our lower bound example)?

- The second problem is whether the technique that we developed for planar 3 -trees can be extended so to improve the upper bound for the queue number of general (that is, non-planar) $k$-trees, which is currently exponential in $k$ [30].

Acknowledgements Open Access funding provided by Projekt DEAL. The authors would like to thank the reviewers of this work for their very helpful comments and suggestions.

Open Access This article is licensed under a Creative Commons Attribution 4.0 International License, which permits use, sharing, adaptation, distribution and reproduction in any medium or format, as long as you give appropriate credit to the original author(s) and the source, provide a link to the Creative Commons licence, and indicate if changes were made. The images or other third party material in this article are included in the article's Creative Commons licence, unless indicated otherwise in a credit line to the material. If material is not included in the article's Creative Commons licence and your intended use is not permitted by statutory regulation or exceeds the permitted use, you will need to obtain permission directly from the copyright holder. To view a copy of this licence, visit http://creativecommons.org/licen ses/by/4.0/.

\section{References}

1. Alam, J.M., Bekos, M.A., Gronemann, M., Kaufmann, M., Pupyrev, S.: Queue layouts of planar 3-trees. In: T.C. Biedl, A. Kerren (eds.) Graph Drawing and Network Visualization, vol. 11282 , LNCS, pp. 213-226. Springer, Berlin (2018). https://doi.org/10.1007/978-3-030-04414-5_15

2. Bekos, M.A., Förster, H., Gronemann, M., Mchedlidze, T., Montecchiani, F., Raftopoulou, C.N., Ueckerdt, T.: Planar graphs of bounded degree have bounded queue number. SIAM J. Comput. 48(5), 1487-1502 (2019). https://doi.org/10.1137/19M125340X

3. Bhatt, S.N., Chung, F.R.K., Leighton, F.T., Rosenberg, A.L.: Scheduling tree-dags using FIFO queues: a control-memory trade-off. J. Parallel Distrib. Comput. 33(1), 55-68 (1996). https://doi. org/10.1006/jpdc.1996.0024

4. Di Battista, G., Eades, P., Tamassia, R., Tollis, I.G.: Graph Drawing: Algorithms for the Visualization of Graphs. Prentice-Hall, New York (1999)

5. Di Battista, G., Frati, F., Pach, J.: On the queue number of planar graphs. SIAM J. Comput. 42(6), 2243-2285 (2013). https://doi.org/10.1137/130908051

6. Di Giacomo, E., Liotta, G., Meijer, H.: Computing straight-line 3D grid drawings of graphs in linear volume. Comput. Geom. 32(1), 26-58 (2005). https://doi.org/10.1016/j.comgeo.2004.11.003

7. Dujmović, V.: Graph layouts via layered separators. J. Comb. Theory Ser. B 110, $79-89$ (2015). https://doi.org/10.1016/j.jctb.2014.07.005

8. Dujmović, V., Frati, F.: Stack and queue layouts via layered separators. J. Graph Algorithms Appl. 22(1), 89-99 (2018). https://doi.org/10.7155/jgaa.00454 
9. Dujmović, V., Joret, G., Micek, P., Morin, P., Ueckerdt, T., Wood, D.R.: Planar graphs have bounded queue-number. In: Zuckerman, D. (ed.) FOCS, pp. 862-875. IEEE Computer Society (2019). https ://doi.org/10.1109/FOCS.2019.00056

10. Dujmović, V., Morin, P., Wood, D.R.: Layout of graphs with bounded tree-width. SIAM J. Comput. 34(3), 553-579 (2005). https://doi.org/10.1137/S0097539702416141

11. Dujmović, V., Pór, A., Wood, D.R.: Track layouts of graphs. Discrete Math. Theoret. Comput. Sci., 6(2), 497-522 (2004). http://dmtcs.episciences.org/315

12. Dujmović, V., Wood, D.R.: Tree-partitions of k-trees with applications in graph layout. In: Bodlaender, H.L. (ed.) WG, vol. 2880 LNCS, pp. 205-217. Springer, Berlin (2003). https://doi. org/10.1007/978-3-540-39890-5_18

13. Dujmović, V., Wood, D.R.: Stacks, queues and tracks: layouts of graph subdivisions. Discrete Math. Theoret. Comput. Sci., 7(1), 155-202 (2005). http://dmtcs.episciences.org/346

14. Felsner, S., Liotta, G., Wismath, S.K.: Straight-line drawings on restricted integer grids in two and three dimensions. J. Gr. Algorithms Appl. 7(4), 363-398 (2003). https://doi.org/10.7155/jgaa.00075

15. Fertin, G., Raspaud, A., Reed, B.A.: On star coloring of graphs. In: Brandstädt, A., Le, V.B. (eds.) WG, vol. 2204 of LNCS, pp. 140-153. Springer, Berlin (2001). https://doi.org/10.1007/3-54045477-2_14

16. Harary, F.: Graph Theory. Addison-Wesley, Reading, MA (1972)

17. Hasunuma, T.: Laying out iterated line digraphs using queues. In: Liotta, G. (ed.) Graph Drawing, volume 2912 of LNCS, pp. 202-213. Springer, Berlin (2003). https://doi.org/10.1007/978-3-54024595-7_19

18. Heath, L.S., Leighton, F.T., Rosenberg, A.L.: Comparing queues and stacks as mechanisms for laying out graphs. SIAM J. Discrete Math. 5(3), 398-412 (1992). https://doi.org/10.1137/0405031

19. Heath, L.S., Rosenberg, A.L.: Laying out graphs using queues. SIAM J. Comput. 21(5), 927-958 (1992). https://doi.org/10.1137/0221055

20. Kaufmann, M., Wagner, D. (eds).: Drawing Graphs, Methods and Models, volume 2025 of LNCS. Springer, Berlin (2001)

21. Mondal, D., Nishat, R.I., Rahman, M.S., Alam, M.J.: Minimum-area drawings of plane 3-trees. J. Gr. Algorithms Appl. 15(2), 177-204 (2011). https://doi.org/10.7155/jgaa.00222

22. Ollmann, T.: On the book thicknesses of various graphs. In Hoffman, F., Levow, R., Thomas, R. (eds.) Southeastern Conference on Combinatorics, Graph Theory and Computing, volume VIII of Congressus Numerantium, p. 459 (1973)

23. Pach, J., Thiele, T., Tóth, G.: Three-dimensional grid drawings of graphs. In Di Battista, G. (ed.) Graph Drawing, volume 1353 of LNCS, pp. 47-51. Springer, Berlin (1997). https://doi. org/10.1007/3-540-63938-1_49

24. Pemmaraju, S.V.: Exploring the powers of stacks and queues via graph layouts. PhD thesis, Virginia Tech (1992)

25. Pupyrev, S.: Mixed linear layouts of planar graphs. In: Graph Drawing, volume 10692 of LNCS, pp. 197-209. Springer, Berlin (2017). https://doi.org/10.1007/978-3-319-73915-1_17

26. Pupyrev, S.: Improved bounds for track numbers of planar graphs. CoRR, 1910.14153, (2019). https ://arxiv.org/abs/1910.14153

27. Rengarajan, S., Madhavan, C.E.V.: Stack and queue number of 2-trees. In: Du, D., Li, M. (eds.) COCOON, volume 959 of LNCS, pp. 203-212. Springer, Berlin (1995). https://doi.org/10.1007/ BFb0030834

28. Shahrokhi, F., Shi, W.: On crossing sets, disjoint sets, and pagenumber. J. Algorithms 34(1), 40-53 (2000). https://doi.org/10.1006/jagm.1999.1049

29. Tarjan, R.E.: Sorting using networks of queues and stacks. J. ACM 19(2), 341-346 (1972). https:// doi.org/10.1145/321694.321704

30. Wiechert, V.: On the queue-number of graphs with bounded tree-width. Electr. J. Comb., 24(1), P1.65, (2017). http://www.combinatorics.org/ojs/index.php/eljc/article/view/v24i1p65

31. Wood, D.R.: Queue layouts, tree-width, and three-dimensional graph drawing. In: Agrawal, M., Seth, A. (eds.) FSTTCS, volume 2556 of LNCS, pp. 348-359. Springer, Berlin (2002). https://doi. org/10.1007/3-540-36206-1_31

32. Yannakakis, M.: Embedding planar graphs in four pages. J. Comput. Syst. Sci. 38(1), 36-67 (1989). https://doi.org/10.1016/0022-0000(89)90032-9 
Publisher's Note Springer Nature remains neutral with regard to jurisdictional claims in published maps and institutional affiliations. 